\title{
Assessment of weight changes during and after pregnancy: practical approaches
}

\author{
Amanda R. Amorim ${ }^{*}$, Yvonne Linné*, Gilberto Kac ${ }^{\ddagger}$ and Paulo M. Lourenço ${ }^{\dagger}$ \\ *Obesity Unit, Karolinska Institutet, Stockholm, Sweden, ${ }^{\dagger}$ Department of Epidemiology, State University of Rio de Janeiro, Rio de Janeiro, Brazil, and \\ ‡Department of Social and Applied Nutrition, Federal University of Rio de Janeiro, Rio de Janeiro, Brazil
}

\begin{abstract}
The usefulness of routine prenatal weight measurements in predicting pregnancy outcomes is still a controversial issue. Comparisons among studies and the interpretation of research findings are complicated due to the variety of indicators applied to express maternal weight changes during and after pregnancy. A review of literature was conducted to clarify the definitions and examine the strengths and limitations of methods for measuring gestational weight gain (WG) and postpartum weight changes. The reasons for weak correlations or non-significant associations between gestational WG and maternal and neonatal outcomes were probably owing to poor quality of obstetrics records and selection of wrong indicators to compute gestational WG. The choice of an indicator depends on clinical and research purpose, availability and reliability of data and cost. Considering the health implication of gestational WG, it is necessary to take into account the measurements used as initial and final weight, accuracy of gestational age estimation and the inclusion of fetal weight as part of maternal WG. Regardless of the indicators used to compute the weight changes after delivery, attention is drawn to the approach for designating prepregnancy weight, the time frame of postpartum weight measurements and the use of overlapping variables, which results in bias (part-whole correlation). It is necessary to address criticisms on the accuracy of prenatal weight measurements and the way of expressing the maternal weight changes during and after pregnancy in order to have reliable results from research.
\end{abstract}

Keywords: pregnancy, postpartum, weight gain, quality of obstetric records.

Correspondence: Amanda R. Amorim, Obesity Unit, Karolinska University Hospital, Huddinge, SE-141 86 Stockholm, Sweden. E-mail: amaamo@ki.se

\section{Introduction}

The clinical utility of gestational weight gain (WG) is still a controversial issue. The growing body of literature indicates that WG during pregnancy is an inexpensive anthropometric indicator to monitor 
maternal and fetal nutritional status, predict pregnancy outcomes and identify women at risk (Institute of Medicine 1990; Parker \& Abrams 1992; Keppel \& Taffel 1993; Schieve et al. 1998; Thorsdottir et al. 2002). However, some authors have argued that the relationship between maternal WG and pregnancy outcomes is not strong enough to make WG a sensitive or specific screening tool for identifying undesirable outcomes (Dawes \& Grudzinskas 1991; Ellison et al. 1997; Honest et al. 2005).

Dawes \& Grudzinskas (1991) showed that among several weight measurements recorded during pregnancy, only maternal weight at booking was significantlyassociatedwithinfantbirthweight. Theauthors advocated that maternal weight needs to be recorded only at first prenatal visit and the subsequent routine weighing of patients may produce unnecessary anxiety and should cease (Dawes \& Grudzinskas 1991). As a result of these findings, British midwives and physicians have changed their attitude towards the usefulness of routinely monitoring WG during gestation (Ellison \& Holliday 1997). Caution is advised in interpreting research results. The possible reasons for weak correlations or non-significant associations between gestational WG and maternal and neonatal outcomes are due to the poor quality of obstetrics records and selection of wrong indicators to compute gestational WG (Ellison \& Holliday 1997; Ellison et al. 1997; Harris et al. 1997).

The variety of indicators applied to express maternal weight changes during and after pregnancy, and the quality of prenatal measurements practice, complicate the interpretation of research findings and comparison between studies. This paper will take particular care in clarifying the definition of gestational WG, postpartum weight retention (PWR), gain and lossinexamining the strengths and limitations ofindicators for measuring maternal weight changes, and their potential use as predictors of pregnancy outcomes. Further, the quality and availability of prenatal weight measurements will be discussed.

\section{Review of literature}

This paper consists of a narrative overview of the literature bearing on anthropometric indicators used to compute maternal weight changes during and after pregnancy. Searches of electronic databases (MEDLINE and LILACS) were conducted to identify the relevant articles. Studies about weighing practice during ante and post-natal care were also considered. Publications written in English, Spanish and Portuguese were included. In addition, the citation lists of relevant publications were searched.

\section{Recommendations of gestational WG}

In 1990, the Institute of Medicine (IOM) published guidelines on gestationalWGbased on prepregnancy body mass index [BMI = weight $(\mathrm{kg}) /$ height $\left.(\mathrm{m})^{2}\right]$. Women with BMIof19.8-26.0shouldgain 11.5-16 kg (25-35 lb) during pregnancy. The target range for desirable gestational WG is higher for underweight womenandlowerforoverweightwomen(Institute of Medicine 1990)

Recommendations for gestational WG vary according to countries. However, since the release of the IOM guidelines, several studies have demonstrated the appropriateness of gaining weight within IOM recommendations for good pregnancy outcomes in different populations (Parker \& Abrams 1992; Keppel \& Taffel 1993; Schieve et al. 1998; Thorsdottir et al.2002; Yekta et al.2006). The literature on this issue shows a clear tendency of IOM recommendationsbeingbroadlyadopted,notonlyin the United States, but also in other countries.

\section{Pattern and composition of gestational WG}

Pregnancy is usually divided into three trimesters: first (less than 14 complete weeks' gestation), second (from the 14th to 27th week gestation), and third (from 28th week until delivery). The WG during the first trimester is relatively small and non-linear. After this period, the rate of gain increases significantly and reach a peak in the second trimester. During the third trimester, the rate slows down slightly, then remains constant until the date of delivery (Institute of Medicine 1990; Abrams et al.1995; Carmichael et al. 1997b). 
Studies concerning the pattern of gestational weight suggested associations with infant outcomes. The studies have found positive relationships between second- or third-trimester WG and birthweight and gestational length (Hickey et al. 1996; Carmichael et al. 1997a). The composition of WG may also affect gestational outcomes (Butte et al. 2003), and distinction between the components of WG should be considered. High rates of gestational WG can be attributed to high fat mass accretion or excessive fluid retention. Each cause is associated with distinct negative outcomes and requires differentclinicalinterventions. The maternalfat accumulation during pregnancy seems to act as a reserve to protect the fetus against nutritional deprivation and ensure lactation after childbirth. Although fat accretion is correlated to fetal size, excessive WG does not greatlyenhancefetalgrowthand pregnancyduration. Instead, it contributes to retention of adipose tissue during postpartum (Lawrence et al.1991; Scholl et al. 1995), which is an important predictor of long-term obesity (Kac et al. 2004; Linne et al. 2004; Rooney et al. 2005).

\section{Gestational WG indicators}

Total weight gain (TWG) is the most common indicator referenced by literature, which is determined by subtracting initial gestational weight from the weight in late pregnancy. The other indicators are: rate of weight gain (RWG), net weight gain (NWG) and net rate of gain (NRG). The RWG is calculated dividing the TWG by gestational age. NWG is defined as the differencebetweenTWGandinfantweight,andNRG is expressed by dividing the NWG by gestational age. Apart from the diversity of indicators, there are also differenttypesofmeasurementsusedtocomposeeach indicator. As an illustration, TWG can be computed using as initial measurement self-reported prepregnancy weight or weight recorded during the first gestational trimester. Weight collected at the day of delivery or at the last prenatal visit can be used as a finalmeasurement.Table 1presentsthedifferentways of measuring and expressing gestational WG.

Decision on which indicator should be applied depends on the outcome of interest. Decision on which measurements should be used relies on the availability and quality of data and particular problems that arise from them.

Taking the association between insufficient WG and low birthweight (LBW) as an example, first, it is essential to bear in mind that LBW can be attributed to prematurity or intrauterine growth restriction (IUGR) (Kramer 1987). Additionally, it is crucial to certify that the WG was calculated considering the last weight measurement during pregnancy, ensuring that women did not gain significant additional weight after that. If the gestational duration was 37 weeks and the total weight was calculated by the 32 nd week, the association could be underestimated because of the interval between the last measurement and the date of delivery. Women may experience additional WG which would not be accounted for. This is of greatconcern, as weight at admission to the maternity unit is not commonly accessible and gestational age at last prenatal visit can be less than gestational age at delivery. Under this circumstance, it is recommended to calculate RWG using gestational age up to the date of last prenatal visit, instead of TWG. Another reason for using RWG is the fact that premature delivery shortens the time of WG. Thus, WG should be expressed independently of the gestational length.

Because the pattern of WG might have an impact on neonatal outcomes (Hickey et al. 1996; Carmichael et al.1997a), RWG, particularly for a specific trimester of gestation, may provide a better understandingoffetalgrowth. However, the datathat are needed to calculate trimester weekly WG are difficult to collect.

In addition to adjustment for gestational duration, it is necessary to separate nutritional and nonnutritionalcontributors, distinguishingthechangesin fat stores or lean body mass from weight increase as a result of increase in the size of fetus, placenta and amniotic fluid (Institute of Medicine 1990). Net maternalWGremovesspuriousassociation related to part-whole correlation, when the weight of the newborn is included in the dependent (birthweight) and independent (maternal WG) variables (Selvin \& Abrams1996; Gunderson\&Abrams1999). TheIOM emphasizes that the use of TWG may provide biased 
Table I. Description of different indicators expressing gestational weight gain and comments on strengths and limitations of measurements used to compose them

\begin{tabular}{ll}
\hline Indicator & Total weight gain (TWG) \\
\hline General formula & TWG = Final weight - Initial weight \\
& - The most commonly used indicator. It is easy to calculate \\
- This indicator is not able to identify the pattern of & maternal WG during pregnancy \\
- Useful to monitor WG because it is computed at the late \\
stage of gestation and intervention cannot be implemented \\
- The total amount of WG depends on gestational length \\
- Used in research and nutritional surveillance
\end{tabular}

\begin{tabular}{|c|c|}
\hline Measurements & $\begin{array}{l}\text { 1. TWG = Final weight - Prepregnancy weight } \\
\text { - Prepregnancy weight is generally difficult to obtain } \\
\text { - Allows to assess the amount of WG since the beginning } \\
\text { of gestation } \\
\text { - Self-reported prepregnancy weight tends to be biased } \\
\text { 2. TWG = Final weight - Weight at first prenatal visit } \\
\text { - Weight at first prenatal visit is commonly available } \\
\text { - Sometimes this measurement cannot be considered as a } \\
\text { proxy of prepregnancy weight } \\
\text { - May affect the calculation of the total amount of WG } \\
\text { when the measurement is recorded after the first trimester } \\
\text { 3. TWG = Weight at delivery - Initial weight } \\
\text { - Weight at delivery is not commonly collected } \\
\text { - Quantifies the total amount of WG during pregnancy } \\
\text { - This information is important for research } \\
\text { 4. TWG = Weight at last prenatal visit - Initial weight } \\
\text { - Weight at last prenatal visit is routinely available } \\
\text { - May underestimate the total amount of WG when the } \\
\text { measurement is collected several weeks before delivery } \\
\text { 5. Adjusted TWG = (Last recorded weight - Prepregnancy } \\
\text { weight) + [Weekly 3rd-trimester weight gain } \times(G A \text { at } \\
\text { delivery - GA at last weight)]* } \\
\text { - Alternative to calculate TWG when GA at last recorded } \\
\text { weight is less than GA at delivery } \\
\text { - Requires information about 3rd-trimester WG } \\
\text { 6. TWG = Self-reported delivery weight - Initial weight } \\
\text { - Is subject to recall bias }\end{array}$ \\
\hline
\end{tabular}

Rate of weight gain (RWG)

RWG $=\left(\right.$ Weight $T_{2}-$ Weight $\left.T_{1}\right) /(G A 2-G A 1)$

- Allows to calculate the velocity of WG between two dates

- This formula does not require prepregnancy weight

- Removes time dependence

- Requires an accurate estimate of gestational length

- Used in research and nutritional surveillance. Potentially useful for screening and clinical practice when the rate is calculated at a stage of pregnancy that allows interventions

1. $\mathrm{RWG}=($ Weight at last recorded 1 st trimester visit Prepregnancy weight)/GA at last recorded 1sttrimester weight*

- Used to calculate the 1 st-trimester weekly WG $\dagger$

- Two measurements of weight at the 1st trimester are not commonly available

2. $\mathrm{RWG}=$ Weight at the end of trimester - Weight at the beginning of trimester/GA at the end of

trimester - GA at the beginning of trimester

- Weight at the beginning and the end of trimester is not commonly available

3. $\mathrm{RWG}=\mathrm{TWG} / \mathrm{GA}$ at the end of gestation

- Assumes linear pattern; however, during the first trimester, WG is non-linear

- GA computed at the end of gestation may not coincide with GA at last recorded weight and hence underestimate the rate

4. $\mathrm{RWG}=\mathrm{TWG} / \mathrm{GA}$ at the last weight

- Assumes linear pattern; however, during the first trimester, WG is non-linear

- Minimize the risk of underestimation of rate results, as it can overestimate the association between insufficient WG and IUGR (Institute of Medicine 1990). Although NWG can remedy this problem, the best solution is to adjust birthweight for gestational age, by dividing the NWG by the length of gestation (NRG).

In spite of the methodological constraints of published data which inflated the association between gestational WG and IUGR, some highquality studies still report a positive correlation of
WG with gestational age-adjusted birthweight (Kramer 1987; Kramer 2003). This suggests that, although overestimation occurs, true association exists.

In contrast, WG seems to have noimpact on length of gestation (Honest et al. 2005). According to Kramer et al. (1992), previous studies suggesting that low gestational WG increases the risk of preterm birth have suffered from several methodological shortcomings, including use of TWG rather than 
Table I. Cont

\begin{tabular}{|c|c|c|}
\hline Indicator & Net weight gain (NWG) & Net rate of gain (NRG) \\
\hline General formula & $\begin{array}{l}\mathrm{NWG}=\mathrm{TWG}-\text { Birthweight } \\
\text { - Measurements are normally available and it is easy to } \\
\text { calculate } \\
\text { - Removes the part-whole correlation with birthweight and } \\
\text { provides maternal WG } \\
\text { - Used in research and nutritional surveillance. However, it } \\
\text { is not useful for clinical practice, as birthweight cannot be } \\
\text { estimated before delivery and corrections cannot be made } \\
\text { during gestation }\end{array}$ & $\begin{array}{l}\mathrm{NRG}=\mathrm{NWG} / \mathrm{GA} \\
\text { - This indicator is not commonly used } \\
\text { - Removes part-whole correlation and time } \\
\text { dependence of WG } \\
\text { - Requires accurate estimation of GA } \\
\text { - Used in research }\end{array}$ \\
\hline Measurements & $\begin{array}{l}\text { 1. NWG = TWG - Weight of products of conception } \\
\text { - Although birthweight is commonly available, the weights } \\
\text { of other components (placenta and amniotic fluid) are not } \\
\text { available } \\
\text { 2. NWG = Postpartum weight - Prepregnancy weight } \\
\text { - Postpartum weight is not often available } \\
\text { - It is time dependent of postpartum weight. Weight } \\
\text { measured immediately after delivery can be influenced by } \\
\text { oedema }\end{array}$ & $\begin{array}{l}\text { 1. } \mathrm{NRG}=\mathrm{NWG} / \mathrm{GA} \text { at the end of gestation } \\
\text { - } \mathrm{GA} \text { at the end of gestation may not coincide with } \\
\mathrm{GA} \text { at last recorded weight and hence underestimate } \\
\text { the rate } \\
\text { 2. } \mathrm{NRG}=\mathrm{NWG} / \mathrm{GA} \text { at the last weight } \\
\text { - Is recommended to use GA at last weight when } \\
\mathrm{NWG} \text { is derived from TWG }\end{array}$ \\
\hline
\end{tabular}

Table adapted from Institute of Medicine (1990). WG, weight gain; T, time; GA, gestational age; *Formula proposed by Nucci et al. (2001); 'The same procedure is applied to calculate 2nd- and 3rd-trimester weekly gain: weight gain between two dates within trimester divided by weeks in the interval of measurements. TWG for trimester can be estimated by multiplying specific trimester weekly weight gain by number of weeks during trimesters: 14 for first and second trimester and 12 for third trimester.

$\mathrm{NRG}$, inclusion of induced preterm deliveries and error-prone gestational age measurements based solely on menstrual dates. The authors attempted to carry out a study, based on obstetric database, to overcome these constraints, which showed that TWG was significantly associated with spontaneous preterm birth. Although the relation persisted when WG was expressed as an overall rate, it disappeared when the analysis was based on NRG (Kramer et al. 1992).

Not only the diversity of WG indicators, but also the variety of unit used to present results may complicate comparison between studies. While some research findings are shown in kilograms, others are in pounds. Further, there are studies that present results which alternate both units. Of course, one unit of weight can be converted to another, but it would be helpful to communicate results in both units or in a consistent way. This practice would improve the clarity of results and enable easy comparison of the magnitude of gestational WG and PWR among studies.

\section{Gestational age}

Although the World Health Organization (WHO) recommends the use of reported last menstrual period (LMP) to estimate gestational age (Alexander et al. 1990), some authors contest that this information might be prone to errors due to irregular cycles, delayed ovulation and memory bias. Apart from that, vaginal bleeding during first trimester of gestation can be confused with last menstruation. Estimation of gestational age based on maternal recollection of the LMP results in misclassification of term, preterm and post-term deliveries and incorrect diagnosis of IUGR. This misclassification can, for example, overestimate the RWG in women, who had delivered at term but whose LMP suggests preterm births and underestimates the rate of gain when women delivered their infants at term, but their LMP dates indicate post-term births. For these reasons, gestational age estimated by early ultrasound examination seems to be more accurate (Kramer et al. 1988; Savitz et al. 2002). 


\section{Techniques for estimating prepregnancy weight}

In compliance with IOM, weight recorded during a recent preconception visit is the best measurement of calculating weight increase during pregnancy (Institute of Medicine 1990). Nevertheless, this information is not frequently available, and weight recorded in early gestation or self-reported weight is often viewed as a proxy of preconception weight.

Maternal weight recorded during the first trimester of gestation is commonly used to estimate gestational WG.Nevertheless, this measurement does not necessarily reflect prepregnancy weight. Even though WG during the first trimester is relatively small, $1-2 \mathrm{~kg}$ (2.2-4.4lb), compared with other periods, inter-individual variation should be considered. Some women are susceptible to nausea and vomiting at early gestation and may experience significant weight loss during this period, while others can gain a considerable amount of weight (Institute of Medicine 1990). Harris \& Ellison (1998) observed mean maternal WG in eight studies that measured bodyweight prior to conception and during early gestation, and showed that WG can range from $-3 \mathrm{~kg}(-6.6 \mathrm{lb})$ at 9 weeks to $+4.5 \mathrm{~kg}(9.9 \mathrm{lb})$ at 15 weeks. Moreover, many women book late into prenatal care, so the first prenatal visit, and therefore the first weight measurement, may not be taken during the first trimester (Chng et al.1980).

Harris et al. $(1997,1998)$ have proposed two alternativestoestimateprepregnancyweight. Thefirstone is based on corrections to adjust weight measured at first trimester. This technique consists of calculating standardized estimates of WG during pregnancy and subtracting the weight gained prior to the first measurement. However, this approach assumes that there is little variation among subjects. The second is based on retrospective extrapolation using two measurements of bodyweight taken early in pregnancy to estimate the bodyweight at conception. The most remarkable limitation of this technique is the usual lack of two measurements of weight during the first trimester (Harris \& Ellison 1998).

Maternal self-reported weight can also be used as proxy of prepregnancy weight. Although selfreportedandmeasurementsofbodyweightarehighly correlated, the accuracy of recalled weight varies with socio-demographic characteristics and BMI. Overweight women tend to underestimate their prepregnancy weight, whereas underweight women tend to overestimate it (Stevens-Simon et al. 1992). Women with ideal bodyweight are also susceptible to misreport their prepregnancy weight owing to errors from inaccurate scales and inadequate weighing practices (Harris et al. 1998).

The errors associated with self-reported weight are diverse: poor weight practice (Ellison \& Holliday 1997), inaccurate scales (Harris et al.1998), and recall and prestige bias, which arises from the desire of women tostate a weight which conforms toperceived norms (Harris \& Ellison 1998). In the face of such limitations, Harris \& Ellison (1998) advocate that self-reported weight is inappropriate for use in research, but is probably adequate for use in clinical settings. In contrast, validation studies showed an error of around $1-2 \mathrm{~kg}(2.2-4.4 \mathrm{lb})$, and stated that self-reported weight is valid to be used in large epidemiological studies (Gunderson et al.2001). As the appropriateness of self-reported weight is still a controversial issue, it is extremely advisable that research validate the prepregnancy weights in a subsample of women before using this measurement in the main study.

Olson \& Strawderman (2003) proposed a procedure to estimate initial weight for women who have started prenatal care in the second trimester. This method is useful when the first prenatal visit occurs after the first trimester of gestation and self-reported weight seems suspect or women do not remember it. Theprocedureforadjustmentconsists of categorizing subjects according to trimester of initial weight measurement. All subjects with measured weights at the first trimester are used to develop a linear regression model. The initial weights for those women joining the study in the second trimester are predicted by the resulting equations based on their first measured weight and their weeks of gestation at the time of entry. In order to evaluate the potential bias of this procedure, the authors compared measures (selfreported weights, weights at early gestation and imputed initial weights), using them to calculate BMI category. Within low, normal and obese determined 
by measured weight, more than $90 \%$ of women selfreported a weight resulting in the same BMI category. However, among those classified in the high-BMI group, $35 \%$ self-reported a weight that they were categorized as normal. Among women with imputed weight, $89 \%$ had agreement between BMI groups calculated from self-reported and imputed weights, with little variation by BMI categories. Due to the bias in self-reported weight in the high-BMI group, the authors decided to use imputed first-trimester weight for determining gestational WG and BMI categoryinstead of recalled prepregnancy weight(Olson \& Strawderman 2003).

\section{Maternal height}

In order to calculate the prepregnancy BMI, it is necessary to obtain information about women's height. Maternal height should be measured at first prenatal visit. Nevertheless, the measurement could be undermined by postural changes if it is taken after 20 weeks of gestation (Institute of Medicine 1990). Selfreported height can also be used, but it is subject to bias. In contrast to weight, height is generally overreported (Stewart 1982; Nawaz et al.2001). The IOM called attention to the negative bias in the selfreported weight, and positive bias in the recalled height can underestimate the BMI to a greater extent (Institute of Medicine 1990).

\section{Pregnancy delivery weight}

With theintention ofminimizingerrorsofestimation, it is possible to define delivery weight as the last weight taken within the 2 weeksimmediately preceding childbirth (Scholl et al. 1995). Another criterion for defining delivery weight is to use measurements taken after 36 weeks' gestation (Fescina 1983). Although after this time point the rate of $\mathrm{WG}$ decreases significantly, when considering post-term pregnancy, theintervalbetweenmeasuredweightand delivery date can be very large, and consequently underestimates the TWG. In this case, the first option seems to be more appropriate. However, maternal weight at 2 weeks before delivery is not usually available.
Because weight at delivery is not frequently measured and weight at last prenatal visit can be taken a long time before childbirth, self-reported pregnancy delivery weight can also be an alternative to calculate WG. Schieve et al. (1999) examined the validity of self-reported delivery weight and found that women reported delivery weight, on average, $0.45 \mathrm{~kg}(0.98 \mathrm{lb})$ less than their measured weight registered in medical records. The magnitude of error is less than reported in validation studies of self-reported prepregnancy weight, but likewise, the accuracy of report varied with socio-demographic characteristics and BMI. The level of underreporting increased significantly with increases in prepregnancy BMI, current BMI, pregnancy WG, and weight change from delivery to recall. The authors used this measurement to calculate and categorize WG, and reported that $30-40 \%$ of women were incorrectly classified, which ultimately might attenuate the association between WG and birthweight (Schieve et al. 1999).

\section{Impact of lack of information on research results}

Another relevant issue is that in studies for calculating gestational WG, women are excluded for not having records of all required weight measurements or due to the lack of information on gestational age at birth. The lack of information is because they usually return infrequently to prenatal visits for further care. It may be that excluded women have lower socioeconomic status and are prone to inadequate gestationalWGandpoorneonatalandmaternaloutcomes. This exclusion could confound the results.

Furthermore, several studies provide no information about the percentage of adolescents included in the sample. Inclusion of adolescents in the analysis may result in inaccurate estimations of $\mathrm{WG}$, because maternal growth can account for weight increase during pregnancy. According to Gunderson \& Abrams (1999), the magnitude of the error depends on the proportion of young teenagers included in the study. In order to have more accurate results, it is advisable to separate adolescents and adults in the analysis. 


\section{Accuracy of prenatal weight measurement}

Bodyweight is subject to variation, owing to the content of bladder and bowel, amount of clothes and shoes worn, and presence of oedema. Weight taken at delivery is especially influenced by status of the membranes, whether or not they have ruptured. The rupture of the membranes results in raginal discharge of amniotic fluid. All these sources of variation enhance the probability of measurement errors (Institute of Medicine 1990, 1992).

Harris et al. (1998) assessed whether the weight data recorded during prenatal care are sufficiently precise for clinical and epidemiological use. The authors investigated the accuracy of weighing equipment and measurements practice recorded in obstetrics notes in 45 prenatal clinics in London. The study showed that beam balance had the highest reliability and validity, whereas scales with spring mechanisms were the least accurate. Nevertheless, only $40 \%$ of clinics surveyed had access to beam balance, and the majority of clinics only had bathroom-type scales with spring mechanisms. It is plausible to think that this situation could be worse in other regions or counties, where healthcare resources are scarce to purchase and maintain accurate weighing equipment. The authors also demonstrated that there was a huge variation of scales in use at the clinics, which suggested that there was a lack of standardization in the type of weighing equipment used and, consequently, extensive variation in the accuracy of maternal weight measurementsrecorded.Most of thematernalweight measurements were inaccurate by around $1-1.5 \%$. Because measurements were taken by trained researchers, the errors were modest. In practice, careless techniques carried out by busy and untrained midwives may be even more inaccurate (Harris et al. 1998).

Despite the fact that inaccuracy may damage the usefulness of weight measurements collected during prenatal care in identifying a high-risk mother and determining the recommended WG during pregnancy, the authors postulated that it is still possible that serial weight measurements recorded on invalid scales can offer a useful measure of WG during preg- nancy, provided that the scales used are highly reliable and the same set of scales are used on each occasion(Harris et al.1998).Notwithstanding,studies reported that prenatal clinics have more than one set of scales that are routinely used; hence it is unlikely that weight measurements of each woman would be recorded always on the same scale (Ellison \& Holliday 1997; Harris et al. 1997; Harris et al. 1998).

Weighing practicewasconsideredinconsistentand careless, in relation to women's position in weighing platform, and removing heavy external clothes or shoes. Weightmeasurementscollectedduringroutine prenatal care are also prone to seasonal changes due to external clothing worn at different stages of pregnancy. Besides that, there is an extensive variation in recording procedures among midwives (Ellison \& Holliday 1997; Harris et al. 1998). The quality of recorded data depends on whether variables were self-reported or directly measured. Self-reported variables were subject to selective omission and subjective bias, whereas measured variables, as previously mentioned, were susceptible to inaccurate equipment and poor measurement practice (Harris et al. 1997).

\section{Definitions of postpartum weight change}

During postpartum, it is possible to calculate three differentindicators:weightretention,WGandweight loss.PWR is defined as the difference between weight at some time after delivery and weight prior to pregnancy. The period of time when postpartum weight is collected varies according to the purposes of studies and availability of measurements. It is possible to find studies reporting weight retention at early postpartum (2-6 weeks after delivery) or 6, 9 and 12 months later. The wide range of time frame complicates the comparison between studies and estimation of the magnitude of weight retention (Gunderson \& Abrams 1999).

Considering the measurements recorded at late stage of postpartum, it is necessary to make a distinction between pregnancies-related weight and additional WG after delivery. Thus, PWG is calculated as the difference between weight at some time after 
delivery and weight immediately after delivery (Gunderson et al. 2001). Negative results from this formula imply weight loss. In this case, this indicator is called, absolute reduction (AR) in weight retention. In addition to this indicator, there are other ways of expressing postpartum weight loss, such as: relative reduction (RR), percentage of weight reduction (\%WR) (Kac et al. 2003), and rate of weight reduction (RWR) (Leermakers et al.1998). The limitations of postpartum weight indicators are the availability of medical records and the difficulty of recruiting postpartum women to research. In order to overcome these drawbacks, some researches use selfreported postpartum weight, which is subject to bias. Tables 2 and 3 provide a description of indicators used to compute PWR and gain/loss, respectively.

Several studies have described that gestational WG has a strong positive correlation with weight retention after delivery (Scholl et al. 1995; Kac et al. 2004; Linne et al.2004). Gestational WG and PWR are normally derived from the same baseline measurements (prepregnancyweight).Therefore,gestationalweight is an intrinsic part of postpartum weight. The use of overlapping variables results in bias due to the partwhole correlation that is similar to correlation betweenTWGandbirthweight.However, thereisnot yet a satisfactory alternative to amend this statistical problem. Although the association is necessarily inflated by using overlapping variables in a linear regression model, it is a matter of fact that correlation between gestational WG and PWR exists, but the magnitude may be smaller than estimated (Gunderson \& Abrams 1999).

Anotherpostpartumweightindicator,proposedby Gunderson et al.(2001),callednetpostpartumweight change, is considered a reasonable option to quantify postpartum WG or loss, when weight at early postpartum is not available. First, it is necessary to calculate the net delivery weight, which is defined as delivery weight (weight at the end of gestation) minus infant birthweight. Then, net postpartum weight change is calculated by subtracting net delivery weight from postpartum weight (Gunderson et al. 2001). It is also possible to calculate net gestational gain using these formulae, by subtracting prepregnancy weight from net delivery weight.

Positive results from the net postpartum weight change formula imply weight gain, and negative results imply weight loss. It is important to emphasize that a negative result does not mean that women did not retain weight. The woman might be slightly slimmer at 6 or 12 months postpartum than immediately

Table 2. Description of the indicator expressing postpartum weight retention and comments on strengths and limitations of measurements used to compose it

\begin{tabular}{|c|c|}
\hline Indicator & Postpartum weight retention (PWR) \\
\hline General formula & $\begin{array}{l}\text { PWR = Postpartum weight - Weight prior to gestation } \\
\text { - Affected by the interval of time between measurements } \\
\text { - Used in research and surveillance }\end{array}$ \\
\hline Measurements & $\begin{array}{l}\text { 1. PWR = Postpartum weight - Reported prepregnancy weight } \\
\text { - Self-reported prepregnancy weight is generally difficult to obtain and tends to be biased } \\
\text { 2. PWR = Postpartum weight }- \text { Weight at first prenatal visit } \\
\text { - Weight at first prenatal visit is commonly available. However, sometimes this measurement cannot be considered as } \\
\text { a proxy of prepregnancy weight } \\
\text { - May underestimate the amount of PWR when measurement is recorded after the first trimester } \\
\text { 3. PWR = Postpartum weight }\left(\mathrm{t}_{0}\right)^{*}-\text { Weight prior to gestation } \\
\text { - Early postpartum weight }\left(\mathrm{t}_{0}\right)^{*} \text { may be affected by oedema } \\
\text { - This measurement is influenced by time since delivery (diereses). Weight measured } 15 \text { days after delivery seems to } \\
\text { be the best time measurement to calculate PWR } \\
\text { 4. PWR = Postpartum weight }\left(\mathrm{t}_{1}\right) \dagger-\text { Weight prior to gestation } \\
\text { - E.g. weight measured } 1 \text { year after delivery. Late postpartum weight may be affected by weight changes (weight } \\
\text { loss/gain) }\end{array}$ \\
\hline
\end{tabular}

*Weight measured immediately after delivery; ${ }^{\dagger}$ Weight collected at some time after delivery. 
Table 3. Description of the indicator expressing postpartum weight gain and loss and comments on their applications

\begin{tabular}{|c|c|}
\hline Indicator & General formula \\
\hline Postpartum weight gain (PWG) & $\begin{array}{l}\text { PWG }=\text { Postpartum weight }\left(\mathrm{t}_{1}\right)^{*} \text { - Postpartum weight }\left(\mathrm{t}_{0}\right) \dagger \\
\text { - Weight after delivery is not often available } \\
\text { - Affected by interval of time between measurements } \\
\text { - Positive results imply weight gain. Negative results indicate weight loss. In this case, the indicator is } \\
\text { called absolute reduction (AR) } \\
\text { - The variants of this indicator are: time when which weight measurement was taken, and use of self- } \\
\text { reported weight, which is subject to recall bias } \\
\text { - Used in research and surveillance }\end{array}$ \\
\hline Absolute reduction (AR) & $\begin{array}{l}\text { AR = Postpartum weight }\left(\mathrm{t}_{1}\right)^{*}-\text { Postpartum weight }\left(\mathrm{t}_{0}\right) \dagger \\
\text { - This indicator has the same characteristics and applications of the previous one (PWG) }\end{array}$ \\
\hline Relative reduction (RR) & $\begin{array}{l}\mathrm{RR}=\left[\text { Postpartum weight }\left(\mathrm{t}_{1}\right)^{*} \text { - Postpartum weight }\left(\mathrm{t}_{0}\right) \dagger\right] / \text { Postpartum weight }\left(\mathrm{t}_{0}\right) \dagger \\
\text { - Affected by the interval of time between early and late postpartum weights } \\
\text { - Used in intervention programmes and research }\end{array}$ \\
\hline $\begin{array}{l}\text { Percentage of weight reduction } \\
\quad(\% \mathrm{WR})\end{array}$ & $\begin{array}{l}\% \mathrm{WR}=\left[\text { Postpartum weight }\left(\mathrm{t}_{1}\right)^{*}-\text { Postpartum weight }\left(\mathrm{t}_{0}\right) \dagger\right] / \mathrm{PWR} \\
\text { - This method indicates the percentage of total amount of weight retained after pregnancy was lost } \\
\text { - } 100 \% \text { indicates that the amount of weight retention was totally lost } \\
\text { - Used in intervention programmes and research }\end{array}$ \\
\hline Rate of weight reduction (RWR) & $\begin{array}{l}\text { RWR }=\left[\text { Postpartum weight }\left(\mathrm{t}_{1}\right) *-\text { Postpartum weight }\left(\mathrm{t}_{0}\right) \dagger\right] / \Delta \mathrm{t} \neq \\
\text { - Denotes the velocity of weight loss } \\
\text { - Used in intervention programmes and research }\end{array}$ \\
\hline
\end{tabular}

*Weight collected at some time after delivery; $†$ Weight measured immediately after delivery; ${ }^{\ddagger}$ Interval between two postpartum measurements in months.

after delivery, but still be over her prepregnancy weight, especially when weight after delivery is derived from mathematical calculations (weight at delivery - birthweight) instead of directly measured.

Because infant birthweight is part of both total gestational gain and early postpartum weight loss, this approach avoids part-whole correlation bias by removing infant birthweight contribution, enabling the assessment of the amount of weight primarily attributed to maternal bodyweight change (Gunderson et al.2001). However, the limitation of this indicator is the scarce availability of weight measurement at delivery and the no consideration of the weight of the products of conception and oedema. Besides that, the formula is a little more complicated than the previous indicators (e.g. PWG or AR).

\section{Conclusion}

To obtain a thorough understanding of the consequence of gestational WG for both mothers and infants, it is crucial to evaluate the total amount of weight gained, the composition and pattern of gain.
Therefore, the interpretation of research results requires careful attention on the definitions of gestational duration, period when weight changes are recorded, and theinclusion or not of newborn weight as part of maternal WG (Institute of Medicine 1990; Harris et al.1998). Clinicians and researchers must be familiar with the different indicators available to compute pregnancy WG and their limitations.

Although measurements of bodyweight recorded during the first trimester or self-reported prepregnancy weight may provide an acceptable indication of preconception weight, the best alternative is to recruit and weigh women before they become pregnant (Harris \& Ellison 1998). It is worth noting that this alternative might be unreasonable, considering the limited financial support given to research. In addition, $28-50 \%$ of pregnancies are unplanned, which restricts prepregnancy recruitment (Henshaw 1998; Matteson et al. 2006; Sedgh et al. 2006). Likewise, although weight measured within 2 weeks of delivery or after 36 weeks' gestation can minimize errors of WGestimations(Institute of Medicine 1992; Scholl et al.1995), the best option still is to encourage 
healthcare providers to measure all parturient women at hospital admission, which is a standard procedure in some countries, for example in Sweden.

Many of the measurements and estimations of weight are subject to errors owing to inaccurate equipment and poor measurement practice and recording procedures. Complex indicators may result in errors of larger magnitude, and these sources of errors probably undermine the utility of weight measurements for clinical and epidemiological use (Ellison \& Holliday 1997; Ellison et al. 1997; Harris et al. 1997, 1998), and may explain why some studies based on obstetrics database found a weak associationbetweengestationalWGandsomeobstetricsand neonatal outcomes. Even though misleading records may underestimate study results, it does not mean that the true association does not exist. Compelling evidences from meta-analysis representing information on over 111000 births in 25 studies from 20 countries indicated the strength of maternal anthropometry in predicting fetal outcomes and its relative weakness in predicting maternal outcomes. Some important maternal outcomes, however, were not assessed, such as: lactation performance, PWR and general morbidity (WHO 1995). It is important to highlight the necessity of adequate weighing equipment and trained healthcare providers capable of measuring and recording accurately changes in maternal bodyweight during and after pregnancy. Elaboration of a meticulous guideline providing information to midwives about the usefulness of weight measurements during prenatal, and on how to measure and register maternal bodyweight changes correctly, might improve the quality and utility of obstetrics records. Although the implementation of weighing training imposes an additional cost to the healthcare system, this training is not onerous (Villar \& Bergsjø2002) compared with the potential benefits of the early identification of women at increased risk of negative pregnancy outcomes.

A number of practical implications can be drawn from this review: (1) beam balances should be preferably used instead of scales with spring mechanisms; (2) women should be weighed without heavy clothes and shoes; (3) bodyweight recorded up to 13 weeks' gestation should be used as an initial weight in the equation of total gestational WG; (4) when lacking information aboutmaternalweight at earlygestation, self-reports can be used after appropriate validation; (5) preferably, weight at delivery should be used as a final measurement; and (6) when delivery weight is not available, maternal weight recorded at 2 weeks before delivery can be used.

In summary, it is necessary to address criticisms on the accuracy of weight measurements during prenatal care and the way of expressing the maternal weight changes during and after pregnancy, in order to have reliable results from research, screening, intervention policy and clinical practices bearing these issues.

\section{Acknowledgements}

This study was supported by a grant from the Brazilian Foundation CAPES for training of researchers in doctorate 'sandwich'. The authors thank A. Adegboye for the constructive criticism and help with grammar and spelling.

\section{References}

Abrams B., Carmichael S. \& Selvin S. (1995) Factors associated with the pattern of maternal weight gain during pregnancy. Obstetrics and Gynecology 86, 170-176.

Alexander G.R., Tompkins M.E. \& Cornely D.A. (1990) Gestational age reporting and preterm delivery. Public Health Reports 105, 267-275.

Butte N.F., Ellis K.J., Wong W.W., Hopkinson J.M. \& Smith E.O. (2003) Composition of gestational weight gain impacts maternal fat retention and infant birth weight. American Journal of Obstetrics and Gynecology 189, 1423-1432.

Carmichael S., Abrams B. \& Selvin S. (1997a) The association of pattern of maternal weight gain with length of gestation and risk of spontaneous preterm delivery. Paediatric and Perinatal Epidemiology 11, 392-406.

Carmichael S., Abrams B. \& Selvin S. (1997b) The pattern of maternal weight gain in women with good pregnancy outcomes. American Journal of Public Health 87, 19841988.

Chng P.K., Hall M.H. \& Macgillivray I. (1980) An audit of antenatal care: the value of the first antenatal visit. British Medical Journal 281, 1184-1186.

Dawes M.G. \& Grudzinskas J.G. (1991) Repeated measurement of maternal weight during pregnancy. Is this a useful practice? British Journal of Obstetrics and Gynaecology 98, 189-194. 
Ellison G.T.H. \& Holliday M. (1997) The use of maternal weight measurements during antenatal care. A national survey of midwifery practice throughout the United Kingdom. Journal of Evaluation in Clinical Practice 3, 30033307.

Ellison G.T.H., Harris H.E. \& Holliday M. (1997) The clinical utility of maternal weight gain as a screen for poor pregnancy outcome. Acta Obstetricia et Gynecologica Scandinavica 76, 51-65.

Fescina R.H. (1983) Aumento de peso durante el embarazo. Metodo para su calculo caundo se desconhece el peso habitual. Boletim de la Oficina Sanitaria Panamericana 95 , 156-161.

Gunderson E.P. \& Abrams B. (1999) Epidemiology of gestational weight gain and body weight changes after pregnancy. Epidemiologic Reviews 21, 261-275.

Gunderson E.P., Abrams B. \& Selvin S. (2001) Does the pattern of postpartum weight change differ according to pregravid body size? International Journal of Obesity and Related Metabolic Disorders 25, 853-862.

Harris H.E. \& Ellison G.T. (1998) Practical approaches for estimating prepregnant body weight. Journal of NurseMidwifery 43, 97-101.

Harris H.E., Ellison G.T., Holliday M. \& Lucassen E. (1997) Methodological considerations in the design of an obstetric database abstracted from medical records. Methods of Information in Medicine 36, 191-200.

Harris H.E., Ellison G.T.H., Holliday M. \& Nickson C. (1998) How accurate are antenatal weight measurements? A survey of hospital and community clinics in a South Thames Region NHS Trust. Paediatric and Perinatal Epidemiology 12, 163-175.

Henshaw S.K. (1998) Unintended pregnancy in the United States. Family Planning Perspectives 30, 24-29.

Hickey C.A., Cliver S.P., Mcneal S.F., Hoffman H.J. \& Goldenberg R.L. (1996) Prenatal weight gain patterns and birth weight among nonobese black and white women Obstetrics and Gynecology 88, 490-496.

Honest H., Bachmann L.M., Ngai C., Gupta J.K., Kleijnen J. \& Khan K.S. (2005) The accuracy of maternal anthropometry measurements as predictor for spontaneous preterm birth - a systematic review. European Journal of Obstetrics and Gynecology and Reproductive Biology 119, 11-20.

Institute of Medicine (1990) Nutrition During Pregnancy: Weight Gain, Nutrient Supplements. National Academy Press: Washington, DC.

Institute of Medicine (1992) Nutrition During Pregnancy and Lactation: An Implementation Guide. National Academy Press: Washington, DC

Kac G., Benicio M.H.A., Valente J.G. \& VelasquezMelendez G. (2003) Postpartum weight retention among women in Rio de Janeiro: a follow-up study. Reports in Public Health 19, 149-161.
Kac G., Benicio M.H.A., Velasquez-Melendez G., Valente J.G. \& Struchiner C.J. (2004) Gestational weight gain and prepregnancy weight influence postpartum weight retention in a cohort of Brazilian women. Journal of Nutrition 134, 661-666.

Keppel K.G. \& Taffel S.M. (1993) Pregnancy-related weight gain and retention: implications of the 1990 Institute of Medicine Guidelines. American Journal of Public Health 83, 1100-1103.

Kramer M.S. (1987) Determinants of low birth weight: methodological assessment and meta-analysis. Bulletin of the World Health Organization 65, 663-737.

Kramer M.S. (2003) The epidemiology of adverse pregnancy outcomes: an overview. Journal of Nutrition 133, 1592S1596S.

Kramer M.S., Mclean F.H., Boyd M.E. \& Usher R.H. (1988) The validity of gestational age estimation by menstrual dating in preterm. term and postterm gestations. Journal of the American Medical Association 22 , 3306-3308

Kramer M.S., Mclean F.H., Eason E.L. \& Usher R.H. (1992) Maternal nutritional and spontaneous preterm birth. American Journal of Epidemiology 136, 574-583.

Lawrence M., Mckillop F.M. \& Durnin J.V.G.A. (1991) Women who gain more fat during pregnancy may not have bigger babies: implications for recommended weight gain during pregnancy. British Journal of Obstetrics and Gynaecology 98, 254-259.

Leermakers E.A., Anglin K. \& Wing R.R. (1998) Reducing postpartum weight retention through a correspondence intervention. International Journal of Obesity and Related Metabolic Disorders 22, 1103-1109.

Linne Y., Dye L., Barkeling B. \& Rossner S. (2004) Longterm weight development in women: a 15-year follow-up of the effects of pregnancy. Obesity Research 12,11661178 .

Matteson K.A., Peipert J.F., Allsworth J., Phipps M.G. \& Redding C.A. (2006) Unplanned pregnancy: does past experience influence the use of a contraceptive method? Obstetrics and Gynecology 107, 121-127.

Nawaz H., Chan W., Abdulrahman M., Larson D. \& Katz D.L. (2001) Self-reported weight and height: implications for obesity research. American Journal of Preventive Med icine 20, 294-298.

Nucci L.B., Ducan B.B., Mengue S.S., Branchtein L., Schmidt M.I. \& Fleck E.T. (2001) Assessment of weight gain during pregnancy in general prenatal care services in Brazil. Cadernos de Saúde Pública 17, 1367-1374.

Olson C.M. \& Strawderman M.S. (2003) Modifiable behavioral factors in a biopsychosocial model predict inadequate and excessive gestational weight gain. Journal of the American Dietetic Association 103, 48-54.

Parker J.D. \& Abrams B. (1992) Prenatal weight gain advice: an examination of the recent prenatal weight rec- 
ommendations of the Institute of Medicine. Obstetrics and Gynecology 79, 664-669.

Rooney B.L., Schauberger C.W. \& Mathiason M.A. (2005) Impact of perinatal weight change on long-term obesity and obesity-related illnesses. Obstetrics and Gynecology 106, 1349-1356.

Savitz D.A., Terry J.W.J., Dole N., Thorp J.M.J., Siega-Riz A.M. \& Herring A.H. (2002) Comparison of pregnancy dating by last menstrual period, ultrasound scanning, and their combination. American Journal of Obstetrics and Gynecology 187, 1660-1666.

Schieve L.A., Cogswell M.E. \& Scanlon K.S. (1998) An empiric evaluation of the Institute of Medicine's pregnancy weight gain guideline by race. Obstetrics and Gynecology 91, 878-884.

Schieve L.A., Perry G.S., Cogswell M.E., Scanlon K.S., Rosenberg D., Carmichael S. et al. (1999) Validity of self-reported pregnancy delivery weight: an analysis of the 1988 National Maternal and Infant Health Survey. American Journal of Epidemiology 150, 947-956.

Scholl T.O., Hediger M.L., Schall J.I., Ances I.G. \& Smith W.K. (1995) Gestational weight gain, pregnancy outcome, and postpartum weight retention. Obstetrics and Gynecology 86, 423-427.

Sedgh G., Bankole A., Oye-Adeniran B., Adewole I.F., Singh S. \& Hussain R. (2006) Unwanted pregnancy and associated factors among Nigerian women. International Family Planning Perspectives 32, 175-184.
Selvin S. \& Abrams B. (1996) Analysing the relationship between maternal weight gain and birthweight: exploration of four statistical issues. Paediatric and Perinatal Epidemiology 10, 220-234.

Stevens-Simon C., Roghmann K.J. \& Mcanarney E.R. (1992) Relationship of self-reported prepregnant weight and weight gain during pregnancy to maternal body habitus and age. Journal of the American Dietetic Association 92 , 85-87.

Stewart A.L. (1982) The reliability and validity of selfreported weight and height. Journal of Chronic Diseases 35, 295-309.

Thorsdottir I., Torfadottir J.E., Birgisdottir B.E. \& Geirsson R.T. (2002) Weight gain in women of normal weight before pregnancy: complications in pregnancy or delivery and birth outcome. Obstetrics and Gynecology 99, 799806.

Villar J. \& Bergsjø P. (2002) New WHO Antenatal Care Model. UNDP/UNFPA/WHO/World Bank Special Programme for Research: Geneva.

World Health Organization (1995) Maternal anthropometry and pregnancy outcomes. A WHO collaborative study. Bulletin of the World Health Organization 73, 1-98.

Yekta Z., Ayatollahi H., Porali R. \& Farzin A. (2006) The effect of pre-pregnancy body mass index and gestational weight gain on pregnancy outcomes in urban care settings in Urmia-Iran. BMC Pregnancy and Childbirth 6, doi:10.1186/1471-2393-6-15. 